\title{
Multi-channel Kalman filters for active noise control
}

\author{
S. van Ophem and A. P. Berkhoff ${ }^{a)}$ \\ University of Twente, Faculty of EEMCS, P.O. Box 217, 7500 AE Enschede, The Netherlands
}

(Received 8 September 2012; revised 29 January 2013; accepted 5 February 2013)

\begin{abstract}
By formulating the feed-forward broadband active noise control problem as a state estimation problem it is possible to achieve a faster rate of convergence than the filtered reference least mean squares algorithm and possibly also a better tracking performance. A multiple input/multiple output Kalman algorithm is derived to perform this state estimation. To make the algorithm more suitable for real-time applications, the Kalman filter is written in a fast array form and the secondary path state matrices are implemented in output normal form. The resulting filter implementation is tested in simulations and in real-time experiments. It was found that for a constant primary path the filter has a fast rate of convergence and is able to track changes in the frequency spectrum. For a forgetting factor equal to unity the system is robust but the filter is unable to track rapid changes in the primary path. A forgetting factor lower than 1 gives a significantly improved tracking performance but leads to a numerical instability for the fast array form of the algorithm.
\end{abstract}

(C) 2013 Acoustical Society of America. [http://dx.doi.org/10.1121/1.4792646]

PACS number(s): 43.50.Ki, 43.60.Ac, 43.60.Mn [SAF]

Pages: $2105-2115$

\section{INTRODUCTION}

The most widely used algorithm for adapting an active noise control (ANC) system is the filtered reference least mean squares algorithm (fx-LMS), due to its simplicity and robustness. One of the biggest drawbacks of the algorithm is its low rate of convergence, resulting in a slow adaption to changes of the primary path. This behavior results from the assumption that the filter coefficients are changing slowly in comparison to the time scale of the plant dynamics, as described, e.g., by Elliott. ${ }^{1}$ To overcome this slow adaption a modified fx-LMS algorithm has been proposed by Bjarnason, ${ }^{2}$ which uses an internal model of the secondary path and the residual error to calculate an estimate of the disturbance.

Recursive least squares (RLS) algorithms have a much faster rate of convergence but require more computational effort. A modified filtered RLS algorithm has been derived by Flockton, ${ }^{3}$ which has a similar structure as the modified fx-LMS algorithm. This algorithm has a few disadvantages: An initial overshoot when the filter is turned on and a slow tracking behavior. Research by Sayed and Kailath ${ }^{4}$ has shown that the RLS filter is just a special case of a Kalman filter. A single input/single output (SISO) augmented Kalman filter was described by Fraanje ${ }^{5}$ in an ANC context, in which it was shown that there is no initial overshoot before convergence when the Kalman filter is properly tuned. This filter estimates the state of the secondary path and the filter coefficients and takes uncertainty of the state and uncertainty in measurements explicitly into account, which explains the absence of the initial overshoot before convergence. Fraanje also establishes a connection between the modified filtered RLS algorithm and the Kalman filter and shows that the modified filtered RLS algorithm is

\footnotetext{
a) Author to whom correspondence should be addressed. Also at: TNO Technical Sciences, Acoustics and Sonar, P.O. Box 96864, 2509 JG The Hague, The Netherlands. Electronic mail: a.p.berkhoff@utwente.nl
}

a special case of the Kalman filter in which the uncertainty of the secondary path estimate is set to zero. The possibility to model system uncertainties in the algorithm leads to a potentially better tracking behavior than the modified filtered RLS algorithm.

In this paper a fast array version of the Kalman filter is derived for a multiple input/multiple output (MIMO) ANC system in Sec. II. An extension of the algorithm with a state space description of the secondary path in output normal form is presented in Sec. III. This description requires less computational effort and is numerically more robust than the full state space description. Its performance is tested in simulations for situations when non-stationary sound sources occur (both sound sources which have changing spectra and moving sound sources) in Secs. IV A to IV D. The validation of the results in real-time experiments in an acoustic lab is described in Sec. IVE.

\section{DERIVATION OF THE MIMO KALMAN FILTER}

\section{A. Model description}

Consider an ANC system which has $N_{x}$ reference channels, $N_{u}$ control channels, and $N_{e}$ error channels with the reference signal vector $\mathbf{x}_{i} \in \mathbb{R}^{N_{x}}$, the control signal vector $\mathbf{u}_{i} \in \mathbb{R}^{N_{u}}$, and the error signal vector $\mathbf{e}_{i} \in \mathbb{R}^{N_{e}}$, in which the subscript $i$ is used as the time index. It is assumed that there is no feedback from the actuators to the reference microphones, so the control system can be seen as a purely feedforward system, as shown in Fig. 1. In this figure $\mathbf{P}(z)$ represents the primary path from the reference microphone to the error microphone, $\mathbf{G}(z)$ represents the secondary path from the secondary actuator to the error microphone, and $\hat{\mathbf{W}}_{i, \mathrm{FIR}}(z)$ is the controller, adapted by a Kalman filter.

The adaptive controller has a feed-forward structure and is described by the matrix $\hat{\mathbf{W}}_{\mathrm{FIR}, i}(z) \in \mathbb{R}^{N_{u} \times N_{x}}$ consisting of FIR-filters with $n_{w}$ filter coefficients. The $(k, l)_{t h}$ term of this matrix, with $0 \leq k \leq N_{u}, 0 \leq l \leq N_{x}$ can be described by 


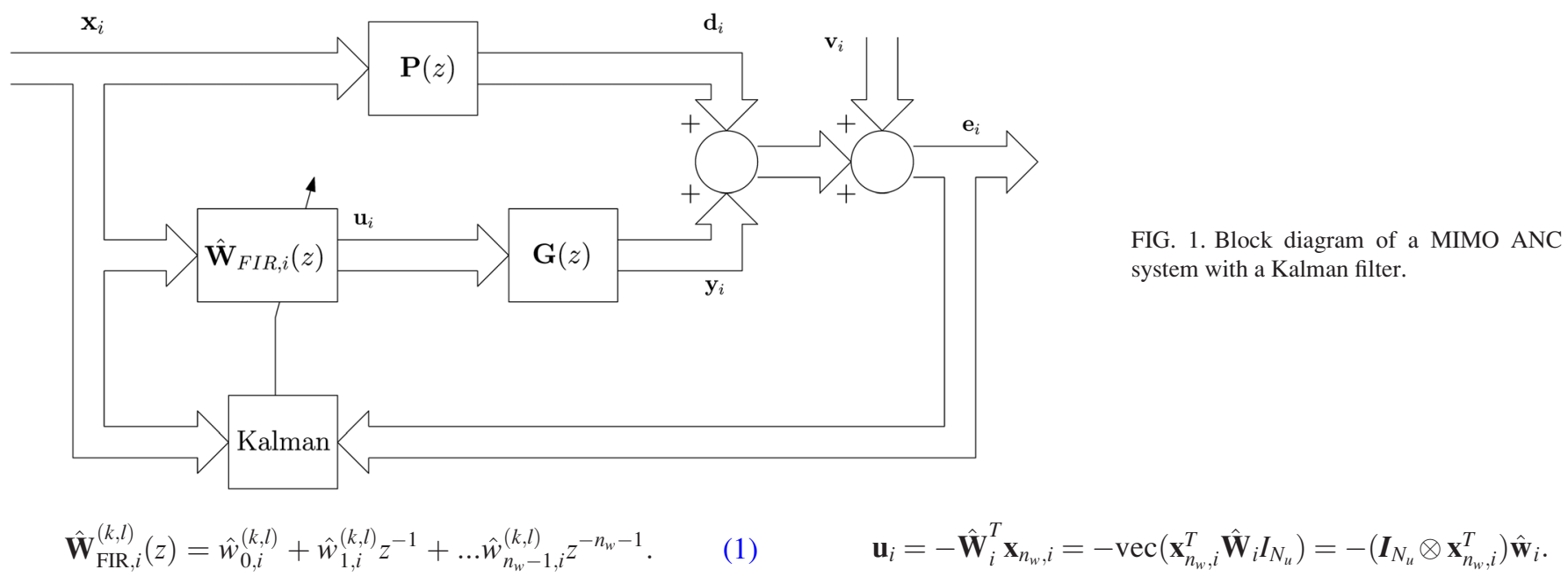

A matrix is derived, which has the individual filter coefficients as entries

$$
\begin{aligned}
& \hat{\mathbf{w}}_{i}^{(k, l)}=\left[\begin{array}{lll}
\hat{w}_{0, i}^{(k, l)} & \ldots & \hat{w}_{n_{w}-1, i}^{(k, l)}
\end{array}\right]^{T} \in \mathbb{R}^{n_{w} \times 1}, \\
& \hat{\mathbf{w}}_{i}^{(k)}=\left[\begin{array}{lll}
\hat{\mathbf{w}}_{i}^{(k, 1)} & \ldots & \hat{\mathbf{w}}_{i}^{\left(k, N_{x}\right)^{T}}
\end{array}\right]^{T} \in \mathbb{R}^{n_{w} N_{x} \times 1}, \\
& \hat{\mathbf{W}}_{i}=\left[\begin{array}{lll}
\hat{\mathbf{w}}_{i}^{(1)} & \ldots & \hat{\mathbf{w}}_{i}^{\left(N_{u}\right)}
\end{array}\right] \in \mathbb{R}^{n_{w} N_{x} \times N_{u}} .
\end{aligned}
$$

The results from Eq. (4) are stacked in a column vector

$$
\hat{\mathbf{W}}_{i}=\operatorname{vec}\left(\hat{\mathbf{W}}_{i}\right) \in \mathbb{R}^{n_{w} N_{u} N_{x}} .
$$

This specific form is needed further below. Also the filter coefficients of the matrix $\mathbf{W}_{i}^{o}(z)$ containing the optimal FIR filters can be written in the same way, giving $\mathbf{w}_{i}^{o(k, l)}, \mathbf{w}_{i}^{o(k)}, \mathbf{W}_{i}^{o}$, and $\mathbf{w}_{i}^{o}$. The $N_{x}$ vectors with the last $n_{w}$ samples of the $N_{x}$ th reference signal $\mathbf{x}_{n_{w}, i}^{N_{x}}$ are stacked in the vector $\mathbf{x}_{n_{w}}$

$$
\begin{aligned}
& \mathbf{x}_{n_{w}, i}^{\left(N_{x}\right)}=\left[\begin{array}{lll}
x_{i}^{\left(N_{x}\right)} & \ldots & x_{i-n_{w}+1}^{\left(N_{x}\right)}
\end{array}\right]^{T} \in \mathbb{R}^{n_{w} \times 1} \\
& \mathbf{x}_{n_{w}}(i)=\left[\begin{array}{llll}
\mathbf{x}_{n_{w}, i}^{(1)^{T}} & \mathbf{x}_{n_{w}, i}^{(2)^{T}} & \ldots & \mathbf{x}_{n_{w}, i}^{\left(N_{x}\right)^{T}}
\end{array}\right]^{T} \in \mathbb{R}^{n_{w} N_{x} \times 1} .
\end{aligned}
$$

The resulting control signals are [using $\operatorname{vec}(\mathbf{A B C})$ $=\left(\mathbf{C}^{T} \otimes \mathbf{A}\right) \operatorname{vec}(\mathbf{B})($ Ref. 6)]

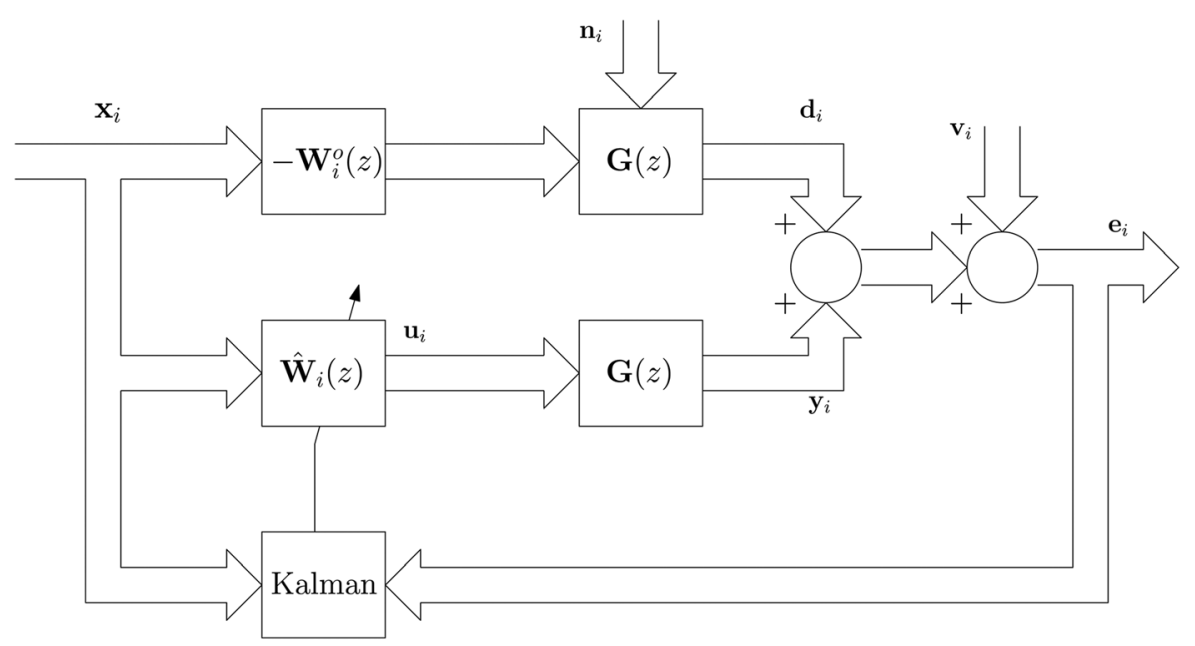

In this equation $\boldsymbol{I}_{N_{u}}$ is the identity matrix of size $N_{u}$ and $\otimes$ is the Kronecker matrix product. When the controller reaches its optimal value $\mathbf{w}_{i}^{o}$, the control signal vector is

$$
\mathbf{u}_{i}^{o}=\left(\boldsymbol{I}_{N_{u}} \otimes \mathbf{x}_{n_{w}, i}^{T}\right) \mathbf{w}_{i}^{o} .
$$

\section{B. Augmented state space description}

The MIMO ANC problem is written in a state space form. The purpose of the FIR controller in series with the secondary path is to minimize the error $\mathbf{e}_{i}=\mathbf{d}_{i}+\mathbf{y}_{i}+\mathbf{v}_{i}$, in which $\mathbf{d}_{i}$ is the influence of the primary path on the error sensors, $\mathbf{y}_{i}$ is the influence of the secondary path on the error sensors, and $\mathbf{v}_{i}$ is assumed to be a Gaussian white noise signal vector, representing measurement noise. The error is minimized when the filter coefficient matrix is adjusted to the optimal value $\mathbf{W}_{i}^{o}(z)$, so that $\mathbf{d}_{i}=-\mathbf{y}_{i}$. Since this situation is achieved when the primary path is equal to the series connection of the optimal FIR filter and the secondary path, the primary path can be approximated by a series connection of the optimal filter coefficients $-\mathbf{W}_{i}^{o}(z)$ and the secondary path $\mathbf{G}(z)$, as stated by Sayyarrodsari et al. ${ }^{7}$ and shown in Fig. 2. A noise vector $\mathbf{n}_{i}$ is included to account for modeling uncertainties.

Suppose the state order of the secondary path is given by $n_{s}$. With these assumptions the primary path can be written in the following state space representation:
FIG. 2. Block diagram of a MIMO ANC system with an approximate representation of the primary path. 


$$
\theta_{i+1}^{(1)}=\boldsymbol{A}_{s} \theta_{i}^{(1)}+\boldsymbol{B}_{s} \mathbf{u}_{i}^{o}+\boldsymbol{H}_{s} \mathbf{n}_{i}, \quad \mathbf{d}_{i}=\boldsymbol{C}_{s} \theta_{i}^{(1)}+\boldsymbol{D}_{s} \mathbf{u}_{i}^{o} .
$$

In these equations the matrices $\boldsymbol{A}_{s}, \boldsymbol{B}_{s}, \boldsymbol{C}_{s}$, and $\boldsymbol{D}_{s}$ are the state matrices of the secondary path, $\boldsymbol{H}_{s} \in \mathbb{R}^{n_{s} \times N_{e} N_{x}}$ is a noise shaping filter, and $\theta_{i}$ is the state vector of the secondary path state. The noise shaping filter can be used if the noise vector $\mathbf{n}_{i}$ is non-white. The series connection of the controller and the secondary path can be written in a state space form in a similar way

$$
\theta_{i+1}^{(2)}=\boldsymbol{A}_{s} \theta_{i}^{(2)}+\boldsymbol{B}_{s} \mathbf{u}_{i}, \quad \mathbf{y}_{i}=\boldsymbol{C}_{s} \theta_{i}^{(2)}+\boldsymbol{D}_{s} \mathbf{u}_{i} .
$$

Since the two state space descriptions have the same state matrices they can be combined, which gives

$$
\begin{aligned}
& \theta_{i+1}=\boldsymbol{A}_{s} \theta_{i}+\boldsymbol{B}_{s}\left(\mathbf{u}_{i}^{o}+\mathbf{u}_{i}\right)+\boldsymbol{H}_{s} \mathbf{n}_{i}, \\
& \mathbf{e}_{i}=\boldsymbol{C}_{s} \theta_{i}+\boldsymbol{D}_{s}\left(\mathbf{u}_{i}^{o}+\mathbf{u}_{i}\right)+\mathbf{v}_{i},
\end{aligned}
$$

with $\theta_{i}=\theta_{i}^{(1)}+\theta_{i}^{(2)}$. By substituting the expressions for the control signals $\mathbf{u}_{i}^{o}$ and $\mathbf{u}_{i}$, derived earlier in Eqs. (8) and (9), an augmented state space description can be derived with the augmented state vector $\chi_{i}=\left[\begin{array}{l}\mathbf{w}_{i} \\ \theta_{i}\end{array}\right]$. When the primary path does not change, the state description of the controller is given by $\mathbf{w}_{i+1}=\mathbf{w}_{i}$ with $\mathbf{w}_{0}=\mathbf{w}_{0}^{o}$. This assumption implies that the Kalman filter will converge to a steady state solution $\mathbf{w}_{i}$. When the optimal filter coefficients change significantly over time, this expression has to be altered to improve tracking. One could consider using an exponential forgetting factor $\lambda$ in the state update equations, such as $\mathbf{w}_{i+1}=\lambda^{-1 / 2} \mathbf{w}_{i}$, in which $0 \ll \lambda \leq 1$. As shown by Sayed and Kailath, ${ }^{4}$ application of a Kalman filter to this state space description leads to the same solution as the exponentially weighted RLS problem. Substitution of Eqs. (8) and (9) and the expression for $\mathbf{w}_{i+1}$ into Eq. (12) leads to the following augmented state space description:

$$
\begin{aligned}
& {\left[\begin{array}{c}
\mathbf{w}_{i+1} \\
\theta_{i+1}
\end{array}\right]=\boldsymbol{A}_{i}\left[\begin{array}{c}
\mathbf{w}_{i} \\
\theta_{i}
\end{array}\right]-\boldsymbol{B}_{i} \hat{\mathbf{w}}_{i}+\boldsymbol{H}_{i} \mathbf{n}_{i},} \\
& \mathbf{e}_{i}=\boldsymbol{C}_{i}\left[\begin{array}{c}
\mathbf{w}_{i} \\
\theta_{i}
\end{array}\right]-\boldsymbol{D}_{i} \hat{\mathbf{w}}_{i}+\mathbf{v}_{i}, \\
& {\left[\begin{array}{c}
\mathbf{w}_{0} \\
\theta_{0}
\end{array}\right]=\left[\begin{array}{c}
\mathbf{w}_{0}^{o} \\
\theta_{0}
\end{array}\right] .}
\end{aligned}
$$

This is the full state space description for a MIMO ANC feed-forward system with the following augmented state matrices:

$$
\begin{aligned}
\mathbf{A}_{i} & =\left[\begin{array}{cc}
\lambda^{-1 / 2} \boldsymbol{I}_{n_{w} N_{u} N_{x}} & 0_{n_{w} N_{u} N_{x} \times n_{s}} \\
\mathbf{B}_{s}\left(\boldsymbol{I}_{N_{u}} \otimes \mathbf{x}_{n_{w}, i}^{T}\right) & \mathbf{A}_{s}
\end{array}\right], \\
\mathbf{B}_{i} & =\left[\begin{array}{c}
0_{n_{w} N_{u} N_{x} \times n_{w} N_{u} N_{x}} \\
-\mathbf{B}_{s}\left(\boldsymbol{I}_{N_{u}} \otimes \mathbf{x}_{n_{w}, i}^{T}\right)
\end{array}\right],
\end{aligned}
$$

$$
\begin{aligned}
& \mathbf{C}_{i}=\left[\mathbf{D}_{s}\left(\boldsymbol{I}_{N_{u}} \otimes \mathbf{x}_{n_{w}, i}^{T}\right) \mathbf{C}_{s}\right], \\
& \mathbf{D}_{i}=-\mathbf{D}_{s}\left(\boldsymbol{I}_{N_{u}} \otimes \mathbf{x}_{n_{w}, i}^{T}\right), \\
& \mathbf{H}=\left[\begin{array}{c}
0_{n_{w} N_{u} N_{x} \times N_{e} N_{x}} \\
\mathbf{H}_{s}
\end{array}\right] .
\end{aligned}
$$

The resulting state space description is given by

$$
\chi_{i+1}=A_{i} \chi_{i}+B_{i} \hat{\mathbf{w}}_{i}+H \mathbf{n}_{i}, \quad \mathbf{e}_{i}=C_{i} \chi_{i}+D_{i} \hat{\mathbf{w}}_{i}+\mathbf{v}_{i} .
$$

\section{Kalman filtering}

To estimate the state of the ANC system a Kalman filter is used. The Kalman filter explicitly takes the covariances from the noise vectors $\mathbf{n}_{i}$ and $\mathbf{v}_{i}$ into account and gives a minimum variance estimate of the state. The initial state is assumed to be uncorrelated with the noise terms $\mathbf{n}_{i}$ and $\mathbf{v}_{i}$, and also the noise terms $\mathbf{n}_{i}$ and $\mathbf{v}_{i}$ are assumed to be uncorrelated with each other, which means

$$
\mathrm{E}\left(\left[\begin{array}{l}
\chi_{0} \\
\mathbf{n}_{0} \\
\mathbf{v}_{0}
\end{array}\right]\left[\begin{array}{l}
\boldsymbol{\chi}_{0} \\
\mathbf{n}_{0} \\
\mathbf{v}_{0}
\end{array}\right]^{T}\right)=\left[\begin{array}{ccc}
\boldsymbol{\Pi}_{0} & 0 & 0 \\
0 & \mathbf{Q} \delta_{k l} & 0 \\
0 & 0 & \mathbf{R} \delta_{k l}
\end{array}\right]
$$

In this equation $\delta_{i j}$ is the Kronecker delta function and $\boldsymbol{\Pi}_{0}$ is called the state covariance matrix, defined as

$$
\boldsymbol{\Pi}_{0}=\left[\begin{array}{cc}
\boldsymbol{\Pi}_{0}^{w w} & \boldsymbol{\Pi}_{0}^{w \theta} \\
\boldsymbol{\Pi}_{0}^{\theta w} & \boldsymbol{\Pi}_{0}^{\theta \theta}
\end{array}\right]
$$

with $\boldsymbol{\Pi}_{0}^{w w}=\mathrm{E}\left[\mathbf{w}_{0} \mathbf{w}_{0}^{T}\right], \boldsymbol{\Pi}_{0}^{w \theta}=\mathrm{E}\left[\mathbf{w}_{0} \theta_{0}^{T}\right]=\boldsymbol{\Pi}_{0}^{\theta w^{T}}, \Pi_{0}^{\theta \theta}=\mathrm{E}\left[\theta_{0} \theta_{0}^{\mathrm{T}}\right]$.

Furthermore, $\mathbf{Q}$ and $\mathbf{R}$ are the noise covariance matrices, and are given by

$$
\begin{gathered}
\mathbf{Q}=\left[\begin{array}{ccc}
Q_{11} & \cdots & 0 \\
\vdots & \ddots & \vdots \\
0 & \ldots & Q_{N_{x} N_{e}}
\end{array}\right], \\
\mathbf{R}=\left[\begin{array}{ccc}
R_{1} & \cdots & 0 \\
\vdots & \ddots & \vdots \\
0 & \cdots & R_{N_{e}}
\end{array}\right] .
\end{gathered}
$$

The MIMO Kalman filter in covariance form is given by the following equations:

$$
\begin{aligned}
& \hat{\chi}_{0}=0_{n_{w} N_{u} N_{x}+n_{s} \times 1}, \\
& \mathbf{P}_{0}=\boldsymbol{\Pi}_{0}, \\
& \boldsymbol{\epsilon}_{i}=\mathbf{e}_{i}-\mathbf{C}_{i} \hat{\chi}_{i}-\mathbf{D}_{i} \hat{\mathbf{w}}_{i}, \\
& \mathbf{R}_{e, i}=\mathbf{R}+\mathbf{C}_{i} \mathbf{P}_{i} \mathbf{C}_{i}^{T}, \\
& \mathbf{K}_{i}=\mathbf{A}_{i} \mathbf{P}_{i} \mathbf{C}_{i}^{T}, \\
& \hat{\chi}_{i+1}=\mathbf{A}_{i} \hat{\chi}_{i}+\mathbf{B}_{i} \hat{\mathbf{w}}_{i}+\mathbf{K}_{i} \mathbf{R}_{e, i}^{-1} \boldsymbol{\epsilon} i, \\
& \mathbf{P}_{i+1}=\mathbf{A}_{i} \mathbf{P}_{i} \mathbf{A}_{i}^{T}-\mathbf{K}_{i} \mathbf{R}_{e, i}^{-1} \mathbf{K}_{i}^{T}+\mathbf{H Q} \mathbf{H}^{T} .
\end{aligned}
$$


A derivation of these equations is given by Sayed. ${ }^{8}$ In these equations $\mathbf{P}_{i}$ represents the covariance matrix of the state estimation error, $\epsilon_{i}$ is the innovation vector, $\mathbf{R}_{e, i}$ is the error covariance matrix, and $\mathbf{K}_{i}$ is the gain matrix. Since the state is augmented, some of these expressions can be reduced and partitioned as follows:

$$
\begin{aligned}
& \mathbf{P}_{i}=\left[\begin{array}{cc}
\mathbf{P}_{i}^{w w} & \mathbf{P}_{i}^{w \theta} \\
\mathbf{P}_{i}^{\theta w} & \mathbf{P}_{i}^{\theta \theta}
\end{array}\right], \quad \mathbf{K}_{i}=\left[\begin{array}{c}
\mathbf{K}_{i}^{w} \\
\mathbf{K}_{i}^{\theta}
\end{array}\right], \\
& \boldsymbol{\epsilon}_{i}=\mathbf{e}_{i}-\mathbf{C}_{s} \hat{\theta}_{i}, \\
& {\left[\begin{array}{c}
\hat{\mathbf{w}}_{i+1} \\
\hat{\theta}_{i+1}
\end{array}\right]=\left[\begin{array}{l}
\hat{\mathbf{w}}_{i} \\
\mathbf{A}_{s} \hat{\theta}_{i}
\end{array}\right]+\left[\begin{array}{c}
\mathbf{K}_{i}^{w} \\
\mathbf{K}_{i}^{\theta}
\end{array}\right] \mathbf{R}_{e, i}^{-1} \boldsymbol{\epsilon}_{i},}
\end{aligned}
$$

in which $\mathbf{K}_{i}^{w} \in \mathbb{R}^{n_{w} N_{u} N_{x} \times N_{e}}$, and $\mathbf{K}_{i}^{\theta} \in \mathbb{R}^{n_{s} \times N_{e}}$. Note that the forgetting factor is not present in Eq. (33), although it would be expected from the definition of Eqs. (14) and (29). The reason for discarding this term is that the filter coefficients would otherwise keep growing when the FIR filter has converged and deviate from the optimal solution. When only forgetting is used in the update equations of the Kalman gain and the Riccati equation, only a change in the FIR filter coefficients would occur if the product $\mathbf{K}_{i}^{w} \mathbf{R}_{e, i}^{-1} \boldsymbol{\epsilon}_{i}$ is large enough, so only when the innovation $\epsilon_{i}$ is becoming too large or when the filter has not converged yet. The straightforward implementation of Eqs. (24) to (30) gives a computationally demanding algorithm.

\section{Shift Invariance}

An algorithm with less floating point operations can be achieved by making use of the shift-invariance of the reference signals. The shift invariance means that for $1 \leq n \leq N_{x}$, the reference signal vector $\mathbf{x}_{n_{w}, i+1}^{(n)}$ is just a shifted version of $\mathbf{x}_{n_{w}, i}^{(n)}$ with one new term. For a multiple channel reference signal this shift behavior can be incorporated by the following equation:

$$
\begin{aligned}
\mathbf{x}_{n_{w}, i}^{T}= & \mathbf{x}_{n_{w}, i+1}^{T}\left(I_{N_{x}} \otimes \mathbf{Z}_{n_{w}}\right) \\
& +\left[\begin{array}{llll}
0_{1 \times n_{w}-1} x_{i-n_{w}+1}^{(1)} & \cdots & 0_{1 \times n_{w}-1} x_{i-n_{w}+1}^{\left(N_{x}\right)}
\end{array}\right] .
\end{aligned}
$$

The matrix $\mathbf{Z}_{n_{w}} \in \mathbb{R}^{n_{w} \times n_{w}}$ is a first diagonal shift matrix. Because of the shift invariance, the state matrices have to be augmented with zeros. The state space matrices become (in which $n_{m}=n_{w} N_{u} N_{x}+N_{u} N_{x}$ is used for a compact notation)

$$
\begin{aligned}
\tilde{\mathbf{A}}_{i} & =\left[\begin{array}{cc}
\lambda^{-1 / 2} \boldsymbol{I}_{n_{m}} & 0_{n_{m} \times n_{s}} \\
\mathbf{B}_{s}\left(\boldsymbol{I}_{N_{u}} \otimes \mathbf{x}_{n_{w}+1, i}^{T}\right) & \mathbf{A}_{s}
\end{array}\right], \\
\tilde{\mathbf{B}}_{i} & =\left[\begin{array}{c}
0_{n_{m} \times n_{m}} \\
-\mathbf{B}_{s}\left(\boldsymbol{I}_{N_{u}} \otimes \mathbf{x}_{n_{w}+1, i}^{T}\right)
\end{array}\right], \\
\tilde{\mathbf{C}}_{i} & =\left[\mathbf{D}_{s}\left(\boldsymbol{I}_{N_{u}} \otimes \mathbf{x}_{n_{w}+1, i}^{T} \mathbf{C}_{s}\right],\right. \\
\tilde{\mathbf{D}}_{i} & =-\mathbf{D}_{s}\left(\boldsymbol{I}_{N_{u}} \otimes \mathbf{x}_{n_{w}+1, i}^{T}\right), \\
\tilde{\mathbf{H}} & =\left[\begin{array}{c}
0_{n_{m} \times N_{x} N_{e}} \\
\mathbf{H}_{s}
\end{array}\right] .
\end{aligned}
$$

The corresponding state space model is

$$
\tilde{\boldsymbol{\chi}}_{i+1}=\tilde{\mathbf{A}}_{i} \tilde{\boldsymbol{\chi}}_{i}+\tilde{\mathbf{B}}_{i} \hat{\mathbf{w}}_{i}+\tilde{\mathbf{H}} \mathbf{n}_{i}, \quad \mathbf{e}_{i}=\tilde{\mathbf{C}}_{i} \tilde{\boldsymbol{\chi}}_{i}+\tilde{\mathbf{D}}_{i} \hat{\mathbf{w}}_{i}+\mathbf{v}_{i} .
$$

The initial state is given by

$$
\begin{aligned}
\tilde{\chi}_{0}= & {\left[\begin{array}{lllll}
\mathbf{w}_{n_{w}, 0}^{(1,1), o^{T}} & 0 & \mathbf{w}_{n_{w}, 0}^{(1,2), o^{T}} & 0 & \ldots \\
& \mathbf{w}_{n_{w}, 0}^{\left(N_{u}, N_{x}\right), o^{T}} & 0 & \theta_{0}^{T}
\end{array}\right]^{T} \in \mathbb{R}^{n_{m}+n_{s} \times 1} . }
\end{aligned}
$$

The state vector $\tilde{\boldsymbol{\chi}}_{i}$ has a similar form since the $N_{\chi} N_{y}\left(n_{w}+1\right)$ th terms for $1 \leq \chi \leq N_{u}$ and $1 \leq y \leq N_{x}$ (the terms which are defined zero) are uncontrollable from the deterministic input $\hat{\mathbf{w}}_{i}$ and the stochastic output $\mathbf{n}_{i}$. This means that they will stay zero for all values of $i \geq 0$

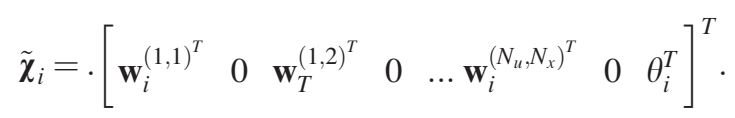

Let us define the vector $\mathbf{w}_{d, i}=\left[\begin{array}{llll}\mathbf{w}_{i}^{(1,1)^{T}} & 0 & \mathbf{w}_{i}^{(1,2)^{T}} & 0\end{array}\right.$ $\left.\ldots \mathbf{w}_{i}^{\left(N_{u}, N_{x}\right)^{T}}\right]^{T} \in \mathbb{R}^{n_{m}-1 \times 1}$, and define the covariance matrix of this vector as $\tilde{\boldsymbol{\Pi}}_{i}^{\mathcal{W}_{d} \boldsymbol{w}_{d}}$. With this definition the covariance matrix of the initial state vector can be written as

$$
\mathrm{E}\left[\tilde{\boldsymbol{\chi}}_{0} \tilde{\boldsymbol{\chi}}_{0}^{T}\right]=\tilde{\boldsymbol{\Pi}}_{0}=\left[\begin{array}{ccc}
\tilde{\boldsymbol{\Pi}}_{0}{ }^{w_{d} w_{d}} & 0_{n_{m}-1 \times 1} & \tilde{\boldsymbol{\Pi}}_{0}^{w_{d} \theta} \\
0_{1 \times n_{m}-1} & 0 & 0_{1 \times n_{s}} \\
\tilde{\boldsymbol{\Pi}}_{0}^{\theta w_{d}} & 0_{n_{s} \times 1} & \tilde{\boldsymbol{\Pi}}_{0}^{\theta \theta}
\end{array}\right] .
$$

To incorporate the shift-invariance property in the Kalman filter equations a pseudo shift-matrix is introduced. The pseudo shift-matrix has the following form:

$$
\boldsymbol{\Psi}=\left[\begin{array}{cc}
\left(\boldsymbol{I}_{N_{u} N_{x}} \otimes \mathbf{Z}_{n_{w}+1}\right) & 0_{n_{m} \times n_{s}} \\
0_{n_{s} \times n_{m}} & \boldsymbol{I}_{n_{s}}
\end{array}\right] .
$$

With this pseudo shift-matrix the relation between the state matrices $\mathbf{A}_{i}, \mathbf{C}_{i}$ and $\mathbf{A}_{i+1}$ and $\mathbf{C}_{i+1}$ can be derived

$$
\begin{aligned}
& \mathbf{x}_{\mathrm{old}, i}=\left[\begin{array}{lllll}
0_{1 \times n_{w}} & x_{i-n_{w}}^{(1)} & 0_{1 \times n_{w}} & \ldots & x_{i-n_{w}}^{\left(N_{x}\right)}
\end{array}\right]^{T}, \\
& \boldsymbol{\Psi} \tilde{\mathbf{A}}_{i}=\tilde{\mathbf{A}}_{i+1} \boldsymbol{\Psi}+\Delta_{i}^{a}, \\
& \tilde{\mathbf{C}}_{i}=\tilde{\mathbf{C}}_{i+1} \boldsymbol{\Psi}+\Delta_{i}^{c}, \\
& \tilde{\mathbf{H}}=\boldsymbol{\Psi} \tilde{\mathbf{H}}, \\
& \Delta_{i}^{a}=\left[\begin{array}{cc}
0_{n_{m} \times n_{m}} & 0_{n_{m} \times n_{s}} \\
\mathbf{B}_{s}\left(\boldsymbol{I}_{N_{u}} \otimes \mathbf{x}_{\mathrm{old}, i}^{T}\right) & 0_{n_{s} \times n_{s}}
\end{array}\right], \\
& \Delta_{i}^{c}=\left[\begin{array}{ll}
\mathbf{D}_{s}\left(\boldsymbol{I}_{N_{u}} \otimes \mathbf{x}_{\mathrm{old}, i}^{T}\right) & 0_{N_{e} \times n_{s}}
\end{array}\right] .
\end{aligned}
$$

The Kalman filter equations for the shift invariant system are the same as stated in Eqs. (24) to (30). The following terms have to be adjusted: 


$$
\begin{gathered}
\overline{\mathbf{K}}_{i}^{w(k)}=\left[\begin{array}{c}
\overline{\mathbf{K}}_{i}^{w(k, 1)} \\
0_{N_{e} \times 1} \\
\overline{\mathbf{K}}_{i}^{w(k, 2)} \\
0_{N_{e} \times 1} \\
\vdots \\
\vdots \\
\overline{\mathbf{K}}_{i}^{w\left(k, N_{x}\right)}
\end{array}\right], \tilde{\mathbf{K}}_{i}=\left[\begin{array}{c}
0_{N_{e} \times 1} \\
\overline{\mathbf{K}}_{i}^{w(1)} \\
\vdots \\
0_{N_{e} \times 1} \\
\overline{\mathbf{K}}_{i}^{w\left(N_{u}\right)} \\
\overline{\mathbf{K}}_{i}^{\theta}
\end{array}\right] . \\
\tilde{\mathbf{P}}_{i}=\left[\begin{array}{ccc}
\tilde{\mathbf{P}}_{i}^{w_{d} w_{d}} & 0_{n_{m}-1 \times 1} & \tilde{\mathbf{P}}_{i}^{w_{d} \theta} \\
0_{1 \times n_{m}-1} & 0 & 0_{1 \times n_{s}} \\
\tilde{\mathbf{P}}_{i}^{\theta w_{d}} & 0_{n_{s} \times 1} & \tilde{\mathbf{P}}_{i}^{\theta \theta}
\end{array}\right],
\end{gathered}
$$

with $\overline{\mathbf{K}}_{i}^{w(k)} \in \mathbb{R}^{\left(n_{w}+1\right) N_{x}-1 \times N_{e}}$, and $\tilde{\mathbf{K}}_{i} \in \mathbb{R}^{n_{m}+n_{s} \times N_{e}}$.

\section{E. Fast array form}

The relations between the augmented state space matrices at instance $i+1$ and $i$, given in Eqs. (46) and (47), can be used to derive a fast array Kalman filter. Array implementations have the following advantages (Sayed and Kailath ${ }^{4}$ ):

(1) Due to the shift-invariance the calculation efficiency is much better than the standard covariance Kalman filter.

(2) The use of square root factors limits the dynamic range.

The reason for the high calculation efficiency of the fast array form of the Kalman filter is that the difference of the Riccati equation is updated instead of the Riccati equation itself

$$
d \tilde{\mathbf{P}}_{i}=\tilde{\mathbf{P}}_{i}-\boldsymbol{\Psi} \tilde{\mathbf{P}}_{i+1} \boldsymbol{\Psi}^{T} .
$$

When proper initial conditions for the covariance matrix are chosen, $d \tilde{\mathbf{P}}_{i}$ can be of very low rank, see Sec. II F. Also the following difference equations are defined:

$$
\begin{gathered}
d \tilde{\mathbf{R}}_{e, i}=\tilde{\mathbf{R}}_{e, i}-\tilde{\mathbf{R}}_{e, i-1}, \\
d \tilde{\mathbf{K}}_{i}=\tilde{\mathbf{K}}_{i}-\boldsymbol{\Psi} \tilde{\mathbf{K}}_{i-1} .
\end{gathered}
$$

Substitution of these variables into the Kalman filter equations leads to

$$
\begin{aligned}
& d \tilde{\mathbf{R}}_{e, i}=\tilde{\mathbf{C}}_{i} d \tilde{\mathbf{P}}_{i} \tilde{\mathbf{C}}_{i}^{T}, \\
& d \tilde{\mathbf{K}}_{i}=\tilde{\mathbf{A}}_{i} d \tilde{\mathbf{P}}_{i} \tilde{\mathbf{C}}_{i}^{T}, \\
& d \tilde{\mathbf{P}}_{i+1}=\tilde{\mathbf{A}}_{i} d \tilde{\mathbf{P}}_{i} \tilde{\mathbf{A}}_{i}^{T}+\Psi \tilde{\mathbf{K}}_{i-1} \tilde{\mathbf{R}}_{e, i-1}^{-1} \tilde{\mathbf{K}}_{i-1}^{T} \Psi^{T}-\tilde{\mathbf{K}}_{i} \tilde{\mathbf{R}}_{e, i}^{-1} \tilde{\mathbf{K}}_{i}^{T} .
\end{aligned}
$$

Assume that $d \tilde{\mathbf{P}}_{i}$ can be factorized as follows (see Sayed and Kailath ${ }^{9}$ ):

$$
d \tilde{\mathbf{P}}_{i}=\tilde{\mathbf{L}}_{i-1} \mathbf{M}_{i-1} \tilde{\mathbf{L}}_{i-1}^{T},
$$

with $\tilde{\mathbf{L}}_{i-1} \in \mathbb{R}^{n_{m}+n_{s} \times \alpha}, \mathbf{M}_{i-1} \in \mathbb{R}^{\alpha \times \alpha}, \alpha \ll n_{m}+n_{s}$, in which $\alpha$ is the rank of matrix $\mathbf{M}_{i-1} \cdot \mathbf{M}_{i-1}$ is a signature matrix of which the exact form will be derived later in Sec. IIF. When this factorization is used the matrices $\tilde{\mathbf{R}}_{e, i-1}, \tilde{\mathbf{K}}_{i-1}$, and $\tilde{\mathbf{L}}_{i-1}$ can be updated with the following fast array algorithm:

$$
\left[\begin{array}{cc}
\tilde{\mathbf{R}}_{e, i-1}^{1 / 2} & \tilde{\mathbf{C}}_{i} \tilde{\mathbf{L}}_{i-1} \\
\boldsymbol{\Psi} \tilde{\mathbf{K}}_{i-1} \tilde{\mathbf{R}}_{e, i-1}^{-T / 2} & \tilde{\mathbf{A}}_{i} \tilde{\mathbf{L}}_{i-1}
\end{array}\right] \boldsymbol{\Theta}_{i-1}=\left[\begin{array}{cc}
\tilde{\mathbf{R}}_{e, i}^{1 / 2} & 0_{N_{e} \times 2 N_{u} N_{x}} \\
\tilde{\mathbf{K}}_{i} \tilde{\mathbf{R}}_{e, i}^{-T / 2} & \tilde{\mathbf{L}}_{i}
\end{array}\right]
$$

The matrix $\boldsymbol{\Theta}_{i-1}$ should be $\boldsymbol{J}$-unitary, in which $\boldsymbol{J}$ is a signature matrix, meaning

$$
\boldsymbol{\Theta}_{i-1} \boldsymbol{J}_{i-1} \boldsymbol{\Theta}_{i-1}^{T}=\boldsymbol{J}_{i-1},
$$

with

$$
\boldsymbol{J}_{i-1}=\left[\begin{array}{cc}
\boldsymbol{I}_{N_{e}} & 0_{1 \times \alpha} \\
0_{\alpha \times 1} & \boldsymbol{M}_{i-1}
\end{array}\right] .
$$

This can be checked by multiplying the left- and right-hand sides of Eq. (33) with $\boldsymbol{J}_{i-1}$ and the transpose of itself. This results in

$$
\begin{aligned}
& {\left[\begin{array}{cc}
\tilde{\mathbf{R}}_{e, i} & \tilde{\mathbf{K}}_{i}^{T} \\
\tilde{\mathbf{K}}_{i} & \tilde{\mathbf{A}}_{i} d \tilde{\mathbf{P}}_{i} \tilde{\mathbf{A}}_{i}^{T}+\boldsymbol{\Psi} \tilde{\mathbf{K}}_{i-1} \tilde{\mathbf{R}}_{e, i-1}^{-1} \tilde{\mathbf{K}}_{i-1}^{T} \boldsymbol{\Psi}^{T}
\end{array}\right]} \\
& =\left[\begin{array}{cc}
\tilde{\mathbf{R}}_{e, i} & \tilde{\mathbf{K}}_{i}^{T} \\
\tilde{\mathbf{K}}_{i} & \tilde{\mathbf{K}}_{i} \tilde{\mathbf{R}}_{e, i}^{T} \tilde{\mathbf{K}}_{i}^{T}+d \tilde{\mathbf{P}}_{i+1}
\end{array}\right]
\end{aligned}
$$

This equation is true when $d \tilde{\mathbf{P}}_{i+1}=\tilde{\mathbf{L}}_{i} \boldsymbol{M}_{i} \tilde{\mathbf{L}}_{i}^{T}$, with $\boldsymbol{M}_{i}$ $=\boldsymbol{M}_{i-1}$. This means we can set, for $i \geq 0$

$$
\boldsymbol{M}=\boldsymbol{M}_{i} \text { and } \boldsymbol{J}=\left[\begin{array}{ll}
\boldsymbol{I}_{N_{e}} & 0_{1 \times \alpha} \\
0_{\alpha \times 1} & \boldsymbol{M}
\end{array}\right]=\boldsymbol{J}_{i} .
$$

It can be concluded that the rank of $d \tilde{\mathbf{P}}$ is dependent on the following initialization of the algorithm:

$$
d \tilde{\mathbf{P}}_{0}=\tilde{\mathbf{L}}_{-1} \boldsymbol{M} \tilde{\mathbf{L}}_{-1}^{T}
$$

With the earlier derived expressions for the gain matrix [Eq. (51)], the updated equations become

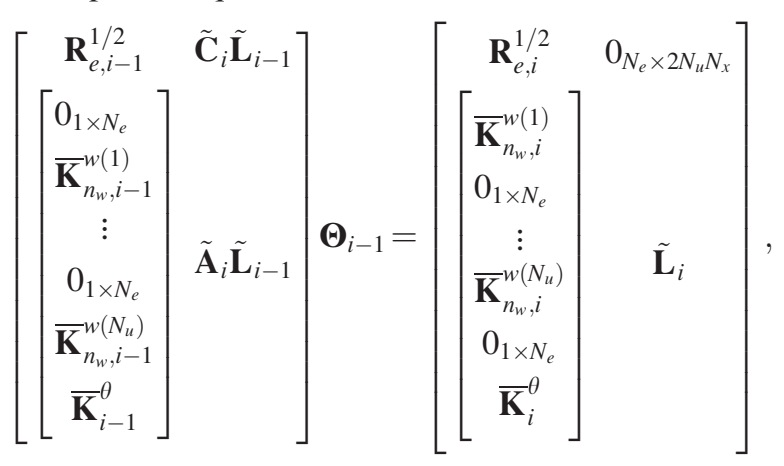

$$
\overline{\mathbf{K}}_{i}^{w}=\left[\begin{array}{c}
\bar{K}_{n_{w}, i}^{w(1,1)} \\
\bar{K}_{n_{w}, i}^{w(1,2)} \\
\vdots \\
\bar{K}_{n_{w}, i}^{w\left(1, N_{x}\right)} \\
\bar{K}_{n_{w}, i}^{w(2,1)} \\
\vdots \\
\vdots \\
\bar{K}_{n_{w}, i}^{w\left(k, N_{x}\right)}
\end{array}\right] .
$$




$$
\left[\begin{array}{c}
\hat{\mathbf{w}}_{n_{w}, i+1} \\
\hat{\theta}_{i+1}
\end{array}\right]=\left[\begin{array}{c}
\hat{\mathbf{w}}_{n_{w}, i} \\
\mathbf{A}_{s} \hat{\theta}_{i}
\end{array}\right]+\left[\begin{array}{c}
\overline{\mathbf{K}}_{i}^{w} \\
\overline{\mathbf{K}}_{i}^{\theta}
\end{array}\right] \mathbf{R}_{e, i}^{-1 / 2} \boldsymbol{\epsilon}_{i},
$$

with $\overline{\mathbf{K}}_{i}^{w} \in \mathbb{R}^{N_{e} \times n_{w} N_{u} N_{x}}$. The transformation matrix $\boldsymbol{\Theta}_{i-1}$ needed to accomplish a $\boldsymbol{J}$-unitary transformation can be achieved by both a combination of a circular Givens rotations and hyperbolic Givens rotations or hyperbolic Householder transformations, as described in Ref. 8.

\section{F. Initialization}

The difference of the Riccati equation must have a reduced rank to make the algorithm more efficient than the covariance form of the Kalman algorithm. By choosing the initial conditions properly, the rank for the Riccati difference equation can be reduced to $\alpha=2 N_{u} N_{x}$. The initialization conditions can be derived with the following equation:

$$
\tilde{\mathbf{P}}_{0}-\boldsymbol{\Psi} \tilde{\mathbf{P}}_{-1} \boldsymbol{\Psi}^{T}=\tilde{\mathbf{L}}_{-1} \boldsymbol{M} \tilde{\mathbf{L}}_{-1}^{T} .
$$

Assume that the reference vector consists only of zeros when the algorithm is initialized: $\mathbf{x}_{n_{w}+1, i}=0_{\left(n_{w}+1\right) N_{x} \times 1}$ for $i \leq-1$. Substitution in the augmented state matrices gives

$$
\begin{aligned}
\tilde{\mathbf{A}}_{-1} & =\left[\begin{array}{cc}
\lambda^{-1 / 2} \boldsymbol{I}_{n_{m}} & 0_{n_{m} \times n_{s}} \\
0_{n_{s} \times n_{m}} & \mathbf{A}_{s}
\end{array}\right], \\
\tilde{\mathbf{C}}_{-1} & =\left[\begin{array}{ll}
0_{1 \times n_{m}} & \mathbf{C}_{s}
\end{array}\right] .
\end{aligned}
$$

Using Eqs. (56) to (58) and Eq. (52) gives

$$
\begin{aligned}
\tilde{\mathbf{P}}_{0} & =\tilde{\mathbf{A}}_{-1} \tilde{\mathbf{P}}_{-1} \tilde{\mathbf{A}}_{-1}^{T}-\tilde{\mathbf{K}}_{-1} \tilde{\mathbf{R}}_{e,-1}^{-1} \tilde{\mathbf{K}}_{-1}^{T}+\tilde{\mathbf{H}} \mathbf{Q} \tilde{\mathbf{H}}^{T} \\
& =\left[\begin{array}{ccc}
\lambda^{-1} \mathbf{P}_{-1}^{w_{d} w_{d}} & 0_{n_{m}-1 \times 1} \lambda^{-1 / 2} \mathbf{P}_{-1}^{w_{d} \theta} \mathbf{A}_{s}^{T} \\
0_{1 \times n_{m}-1} & 0 & 0_{1 \times n_{s}} \\
\lambda^{-1 / 2} \mathbf{A}_{s} \mathbf{P}_{-1}^{\theta w_{d}} & 0_{n_{s} \times 1} & \mathbf{A}_{s} \mathbf{P}_{-1}^{\theta \theta} \mathbf{A}_{s}^{T}
\end{array}\right] \\
& -\left[\begin{array}{c}
\lambda^{-1 / 2} \mathbf{P}_{-1}^{w_{d} \theta} \mathbf{C}_{s}^{T} \\
0 \\
\mathbf{A}_{s} \mathbf{P}_{-1}^{\theta \theta} \mathbf{C}_{s}^{T}
\end{array}\right]\left(\mathbf{R}+\mathbf{C}_{s} \mathbf{P}_{-1}^{\theta \theta} \mathbf{C}_{s}^{T}\right)^{-1}\left[\begin{array}{c}
\lambda^{-1 / 2} \mathbf{P}_{-1}^{w_{d} \theta} \mathbf{C}_{s}^{T} \\
0 \\
\mathbf{A}_{s} \mathbf{P}_{-1}^{\theta \theta} \mathbf{C}_{s}^{T}
\end{array}\right] \\
& +\left[\begin{array}{ccc}
0_{n_{m}-1 \times n_{m}-1} & 0_{n_{m}-1 \times 1} 0_{n_{m}-1 \times n_{s}} \\
0_{1 \times n_{m}-1} & 0 & 0_{1 \times n_{s}} \\
0_{n_{s} \times n_{m}-1} & 0_{n_{s} \times 1} & \mathbf{H}_{s} \mathbf{Q} \mathbf{H}_{s}^{T}
\end{array}\right] .
\end{aligned}
$$

The sub-matrices $1-2$ and $2-1$ of $\tilde{\mathbf{P}}_{-1}$ are set to zero (assuming the filter coefficients and the secondary path states are uncorrelated)

$$
\begin{aligned}
\mathbf{P}_{-1} & =\left[\begin{array}{cc}
\boldsymbol{\Pi}_{-1}^{w w} & 0_{n_{w} N_{u} N_{x} \times n_{s}} \\
0_{n_{s} \times n_{w} N_{u} N_{x}} & \boldsymbol{\Pi}_{-1}^{\theta \theta}
\end{array}\right] \\
& =\left[\begin{array}{cc}
\mathbf{P}_{-1}^{w w} & 0_{n_{w} N_{u} N_{x} \times n_{s}}^{w w} \\
0_{n_{s} \times n_{w} N_{u} N_{x}} & \mathbf{P}_{-1}^{\theta \theta}
\end{array}\right] .
\end{aligned}
$$
giving

Eqs. (72) and (73) can now be substituted into Eq. (69),

$$
\tilde{\mathbf{P}}_{0}-\boldsymbol{\Psi} \tilde{\mathbf{P}}_{-1} \boldsymbol{\Psi}^{T}=\left[\begin{array}{ll}
d \tilde{P}_{11} & d \tilde{P}_{12} \\
d \tilde{P}_{21} & d \tilde{P}_{22}
\end{array}\right]
$$

with

$$
\begin{aligned}
d \tilde{P}_{11}= & \lambda^{-1}\left[\begin{array}{cc}
\boldsymbol{\Pi}_{-1}^{w_{d} w_{d}} & 0_{n_{m}-1 \times 1} \\
0_{1 \times n_{m}-1} & 0
\end{array}\right] \\
& -\left(\boldsymbol{I}_{N_{x}} \otimes \mathbf{Z}_{n_{w}}\right)\left[\begin{array}{cc}
\boldsymbol{\Pi}_{-1}^{w_{d} w_{d}} & 0_{n_{m}-1 \times 1} \\
0_{1 \times n_{m-1}} & 0
\end{array}\right] \\
& \times\left(\boldsymbol{I}_{N_{x}} \otimes \mathbf{Z}_{n_{w}}\right)^{T}, \\
d \tilde{P}_{12}= & 0_{n_{m} \times n_{s}}, \\
d \tilde{P}_{21}= & 0_{n_{s} \times n_{m}}, \\
d \tilde{P}_{22}= & \mathbf{A}_{s} \boldsymbol{\Pi}_{-1}^{\theta \theta} \mathbf{A}_{s}^{T}-\mathbf{A}_{s} \boldsymbol{\Pi}_{-1}^{\theta \theta} \mathbf{C}_{s}^{T}\left(\mathbf{R}+\mathbf{C}_{s} \boldsymbol{\Pi}_{-1}^{\theta \theta} \mathbf{C}_{s}^{T}\right)^{-1} \\
& \times \mathbf{C}_{s} \boldsymbol{\Pi}_{-1}^{\theta \theta} \mathbf{A}_{s}^{T}+\mathbf{H}_{s} \mathbf{Q} \mathbf{H}_{s}^{T}-\boldsymbol{\Pi}_{-1}^{\theta \theta} .
\end{aligned}
$$

Let us choose

$$
\mathbf{P}_{-1}^{w w}=\left(\sqrt{\delta} \boldsymbol{I}_{N_{u} N_{x}}\right) \otimes \operatorname{diag}\left\{\lambda, \lambda^{2}, \ldots, \lambda^{n_{w}}\right\},
$$

in which $\delta$ is a regularization factor. With this expression it is possible to rewrite $d \tilde{P}_{11}$

$$
\begin{aligned}
d \tilde{P}_{11}= & \lambda^{-1}\left[\begin{array}{cc}
\Pi_{-1}^{w_{d} w_{d}} & 0_{n_{m}-1 \times 1} \\
0_{1 \times n_{m}-1} & 0
\end{array}\right]-\left(I_{N_{x} N_{u}} \otimes \mathbf{Z}_{n_{w}+1}\right) \\
& \times\left[\begin{array}{cc}
\Pi_{-1}^{w_{d} w_{d}} & 0_{n_{m}-1 \times 1} \\
0_{1 \times n_{m}-1} & 0
\end{array}\right]\left(\mathbf{I}_{N_{x} N_{u}} \otimes \mathbf{Z}_{n_{w}+1}\right)^{T}
\end{aligned}
$$

which yields

$$
d \tilde{P}_{11}=\delta\left(\boldsymbol{I}_{N_{u} N_{x}} \otimes\left[\begin{array}{ccc}
1 & 0_{1 \times n_{w}-1} & 0 \\
0_{n_{w}-1 \times 1} & 0_{n_{w}-1 \times n_{w}-1} & 0_{n_{w}-1 \times 1} \\
0 & 0_{1 \times n_{w}-1} & -\lambda^{n_{w}}
\end{array}\right]\right) .
$$

If $\left(\mathbf{A}_{s}, \mathbf{C}_{s}\right)$ is detectable and $\left(\mathbf{A}_{s}, \mathbf{H}_{s} \mathbf{Q}^{1 / 2}\right)$ is stabilizable, then there exists a solution $\boldsymbol{\Pi}_{-1}^{\theta \theta}$ for the discrete algebraic Riccati equation (see Ref. 10, theorem 6.2.).

$$
\begin{aligned}
\mathbf{A}_{s} \boldsymbol{\Pi}_{-1}^{\theta \theta} \mathbf{A}_{s}^{T}-\mathbf{A}_{s} \boldsymbol{\Pi}_{-1}^{\theta \theta} \mathbf{C}_{s}^{T}\left(\mathbf{R}+\mathbf{C}_{s} \boldsymbol{\Pi}_{-1}^{\theta \theta} \mathbf{C}_{s}^{T}\right)^{-1} \mathbf{C}_{s} \boldsymbol{\Pi}_{-1}^{\theta \theta} \mathbf{A}_{s}^{T} \\
\quad+\mathbf{H}_{s} \mathbf{Q} \mathbf{H}_{s}^{T}-\boldsymbol{\Pi}_{-1}^{\theta \theta}=0_{n_{s} \times n_{s}} .
\end{aligned}
$$

When these solutions are substituted into the Riccati difference and the solution is decomposed, this gives

$$
\begin{aligned}
& \tilde{\mathbf{L}}_{-1}=\sqrt{\delta}\left[\boldsymbol{I}_{N_{u} N_{x}} \otimes\left[\begin{array}{cc}
1 & 0 \\
0_{n_{w}-1 \times 1} & 0_{n_{w}-1 \times 1} \\
0 & \lambda^{n_{w} / 2}
\end{array}\right],\right. \\
& \boldsymbol{M}=\boldsymbol{I}_{N_{u} N_{x}} \otimes\left[\begin{array}{cc}
1 & 0 \\
0 & -1
\end{array}\right],
\end{aligned}
$$




$$
\boldsymbol{J}=\left(\boldsymbol{I}_{N_{e}} \oplus \boldsymbol{M}\right)=\left(\boldsymbol{I}_{N_{e}} \oplus\left(\boldsymbol{I}_{N_{u} N_{x}} \otimes\left[\begin{array}{cc}
1 & 0 \\
0 & -1
\end{array}\right]\right)\right) .
$$

In Eq. (85), $\oplus$ is a matrix sum operator. Also the following initial conditions are needed to start the algorithm:

$$
\begin{aligned}
\mathbf{R}_{e,-1}^{1 / 2} & =\left(\mathbf{R}+\mathbf{C}_{s} \boldsymbol{\Pi}_{-1}^{\theta \theta} \mathbf{C}_{s}^{T}\right)^{1 / 2} \in \mathbb{R}^{N_{e} \times N_{e}}, \\
\overline{\mathbf{K}}_{-1}^{w} & =0_{n_{w} N_{u} N_{x} \times N_{e}}, \\
\overline{\mathbf{K}}_{-1}^{\theta} & =\mathbf{A}_{s} \boldsymbol{\Pi}_{-1}^{\theta \theta} \mathbf{C}_{s}^{T} \mathbf{R}_{e, i}^{-1 / 2} .
\end{aligned}
$$

\section{OUTPUT NORMAL FORM PARAMETERIZATION}

The state space description of the secondary path is rewritten to an output normal form parameterization. This parameterization has advantages in comparison to the full state space model. As shown in Ref. 11, not only a reduction of floating point operations is achieved by transforming to the Hessenberg form of the state matrix but the parameterization also makes it possible to solve the floating point operations in a recursive way, leading to even more reduction of the floating point operations needed.

Another benefit of the state space parameterization is the removal of redundancy in the state matrices. The removal of redundancy can make the filter less sensitive to small changes in the secondary transfer function path.

\section{A. Transformation to output normal form}

When the secondary path does not change significantly over time, the state matrices can be identified off-line. After identification the states can be transformed to the output normal form if the states are observable. The transformation is done in such a way that the observability Gramian of the state matrices $\mathbf{A}_{s}$ and $\mathbf{C}_{s}$ is the identity matrix

$$
\mathbf{A}_{s}^{T} \mathbf{A}_{s}+\mathbf{C}_{s}^{T} \mathbf{C}_{s}=\boldsymbol{I} .
$$

This transformation gives orthogonal states, with several numerical advantages in comparison to the full state space model, in which the most important are the low round off noise gain and the notion that the amplitude of the signal is not changed throughout the filter (Roberts and Mullis ${ }^{12}$ ). The transformation of the state space model is done with a similarity transform matrix $\boldsymbol{T}_{t}$. This matrix can be determined by calculating the solution $\boldsymbol{Z}$ from the observability Gramian of the full state space system $\mathbf{A}_{s}^{T} \boldsymbol{Z} \mathbf{A}_{s}+\mathbf{C}_{s}^{T} \mathbf{C}_{s}=\boldsymbol{Z}$, by decomposing this solution $\left(\boldsymbol{Z}=\boldsymbol{T}_{z} \boldsymbol{T}_{z}^{T}\right)$ and calculating $\boldsymbol{T}_{t}=\boldsymbol{T}_{z}^{-T}$. The state matrices in output normal form can be calculated with $\mathbf{A}_{T}=\boldsymbol{T}_{t} \mathbf{A} \boldsymbol{T}_{t}^{-1}, \mathbf{B}_{T}=\boldsymbol{T}_{t} \mathbf{B}, \mathbf{C}_{T}=\mathbf{C} \boldsymbol{T}_{t}^{-1}$, and $\mathbf{D}_{T}=\mathbf{D}$. When these transformations are done, the columns of the matrix $\left[\begin{array}{l}\mathbf{C}_{T} \\ \mathbf{A}_{T}\end{array}\right]$ are orthogonal. A second similarity transformation is done to transform the matrix to a Hessenberg form (the required transformation matrix can be calculated with Givens rotations or Householder transformations). The resulting matrix $\left[\begin{array}{l}\mathbf{C}_{H} \\ \mathbf{A}_{H}\end{array}\right]$ can now be decomposed with the following parameterization:

$$
\left[\begin{array}{l}
\mathbf{C}_{H} \\
\mathbf{A}_{H}
\end{array}\right]=\boldsymbol{T}_{1}(\beta(1)) \ldots \boldsymbol{T}_{n}(\beta(n))\left[\begin{array}{l}
0 \\
\boldsymbol{I}_{n}
\end{array}\right] .
$$

In this equation $\beta_{A C}=\left[\begin{array}{lll}\beta(1) & \ldots & \beta(n)\end{array}\right]^{T}$ is a vector with parameters ranging from -1 to 1 and $\boldsymbol{T}_{1} \ldots . . \boldsymbol{T}_{n}$ are rotation matrices. Their definitions are given in Ref. 11.

\section{B. Incorporation into the Kalman filter}

To incorporate the output normal form parameterization into the ANC Kalman filter in fast array form, a few adjustments have to be made since the parameterization is only useful for the part of the augmented state matrices that represent the secondary path. The matrix $\tilde{\mathbf{L}}_{i-1}$ has to be partitioned in the following way:

$$
\tilde{\mathbf{L}}_{i-1}=\left[\begin{array}{c}
\tilde{\mathbf{L}}_{p_{1}, i-1} \\
\tilde{\mathbf{L}}_{p_{2}, i-1}
\end{array}\right]
$$

in which $\tilde{\mathbf{L}}_{p_{1}, i-1} \in \mathbb{R}^{n_{m} \times 2 N_{u} N_{x}}$, and $\tilde{\mathbf{L}}_{p_{2}, i-1} \in \mathbb{R}^{n_{s} \times 2 N_{u} N_{x}}$. The parameterization can now be used to calculate

$$
\left[\begin{array}{l}
\mathbf{C}_{H} \\
\mathbf{A}_{H}
\end{array}\right] \tilde{\mathbf{L}}_{p_{2}, i-1}=\boldsymbol{T}_{1}(\beta(1)) \ldots . \boldsymbol{T}_{n}(\beta(n))\left[\begin{array}{c}
0 \\
\tilde{\mathbf{L}}_{p_{2}, i-1}
\end{array}\right]
$$

Since the calculation of $\mathbf{C}_{i} \tilde{\mathbf{L}}_{i-1}$ also requires $\tilde{\mathbf{L}}_{p_{1}, i-1}$, the end result is calculated with

$$
\mathbf{C}_{i} \tilde{\mathbf{L}}_{i-1}=\mathbf{D}_{s}\left(\boldsymbol{I}_{N_{u}} \otimes \mathbf{x}_{n_{w}+1, i}^{T}\right) \tilde{\mathbf{L}}_{p_{1}, i-1}+\mathbf{C}_{H} \tilde{\mathbf{L}}_{p_{2}, i-1}
$$

Also the state matrix $\mathbf{A}_{i}$ has to be partitioned into $\mathbf{A}_{i}$ $=\left[\begin{array}{l}\mathbf{A}_{p_{1}, i} \\ \mathbf{A}_{p_{2}, i}\end{array}\right]$ with $\quad \mathbf{A}_{p_{1}, i} \in \mathbb{R}^{n_{m} \times n_{m}+n_{s}}$ and $\quad \mathbf{A}_{p_{2}, i} \in \mathbb{R}^{n_{s} \times n_{m}+n_{s}}$. The matrix multiplication $\mathbf{A}_{i} \tilde{\mathbf{L}}_{i-1}$ can now be executed with

$$
\begin{aligned}
\mathbf{A}_{p_{1}, i} \tilde{\mathbf{L}}_{i-1} & =\left[\begin{array}{ll}
\lambda^{-1 / 2} \boldsymbol{I}_{n_{m}} & 0_{n_{m} \times n_{s}}
\end{array}\right] \tilde{\mathbf{L}}_{i-1}, \\
\mathbf{A}_{p_{2}, i} \tilde{\mathbf{L}}_{i-1} & =\mathbf{B}_{s}\left(\boldsymbol{I}_{N_{u}} \otimes \mathbf{x}_{n_{w}}^{T}(i)\right) \tilde{\mathbf{L}}_{p_{1}, i-1}+\mathbf{A}_{H} \tilde{\mathbf{L}}_{p_{2}, i-1} .
\end{aligned}
$$

Also the update equations can be rewritten to a more efficient form by using a dummy update vector:

$$
\begin{aligned}
& {\left[\begin{array}{l}
\hat{\mathbf{e}}_{d, i} \\
\hat{\mathbf{x}}_{d, i}
\end{array}\right]=\left[\begin{array}{c}
\mathbf{C}_{H} \\
\mathbf{A}_{H}
\end{array}\right] \hat{\theta}_{i}=\boldsymbol{T}_{1}(\beta(1)) \ldots \boldsymbol{T}_{n}(\beta(n))\left[\begin{array}{c}
0 \\
\hat{\theta}_{i}
\end{array}\right],} \\
& \epsilon_{i}=\mathbf{e}_{i}-\hat{\mathbf{e}}_{d, i} \in \mathbb{R}^{N_{e} \times 1}, \\
& \hat{\theta}_{i+1}=\hat{\mathbf{x}}_{d, i}+\overline{\mathbf{K}}_{i}^{\theta} \mathbf{R}_{e, i}^{-1 / 2} \epsilon_{i} \in \mathbb{R}^{n_{s} \times 1} .
\end{aligned}
$$




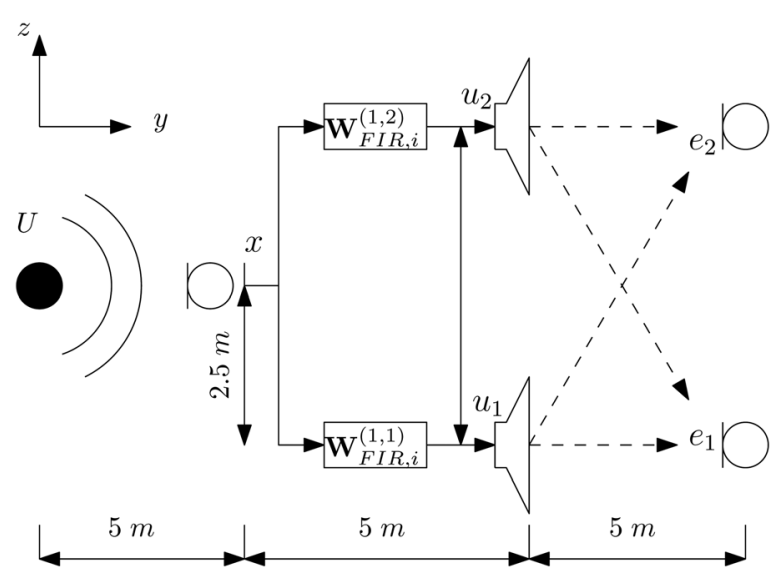

FIG. 3. MIMO setup.

When these alternate equations are used, only the parameters $\beta_{A C}=\left[\begin{array}{lll}\beta(1) & \ldots & \beta(n)\end{array}\right]^{T}$ are needed for the state updates instead of the state matrices $\mathbf{A}_{s}$ and $\boldsymbol{C}_{s}$.

\section{RESULTS}

The performance of the derived algorithm was tested in simulations and in a real-time ANC application. In Sec. IV A the results of a stationary MIMO setup are shown. In Sec. IV B the same setup with a changing spectrum is shown. In Sec. IV C the results of a system with a moving sound source are shown. The simulation results are compared to real-time experimental results of a feed-forward ANC system in a duct in Sec. IV E.

\section{A. Stationary MIMO setup}

A MIMO setup as depicted in Fig. 3 was used to analyze the performance of the algorithm for a stationary noise source. The setup has one reference sensor $x$, two error sensors $e_{1}$ and $e_{2}$, and two secondary sources driven by the control signals $u_{1}$ and $u_{2}$. At the left-hand side a primary noise source $U$ is positioned, which emits a white noise signal. The primary and secondary sources are point, monopole
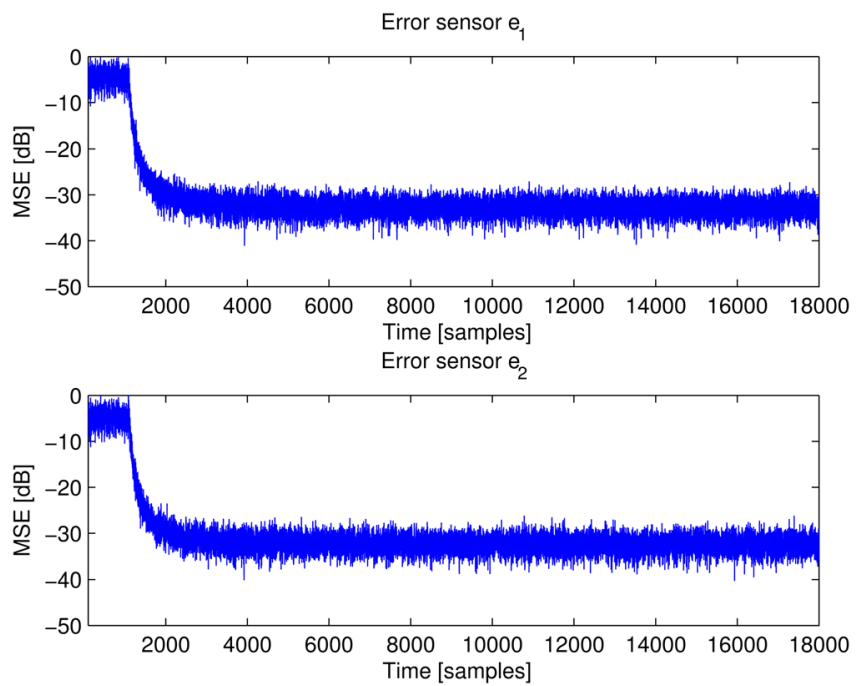

FIG. 4. (Color online) Mean squared error at the error sensors (filter turned on after 1000 samples, average of 50 simulations).

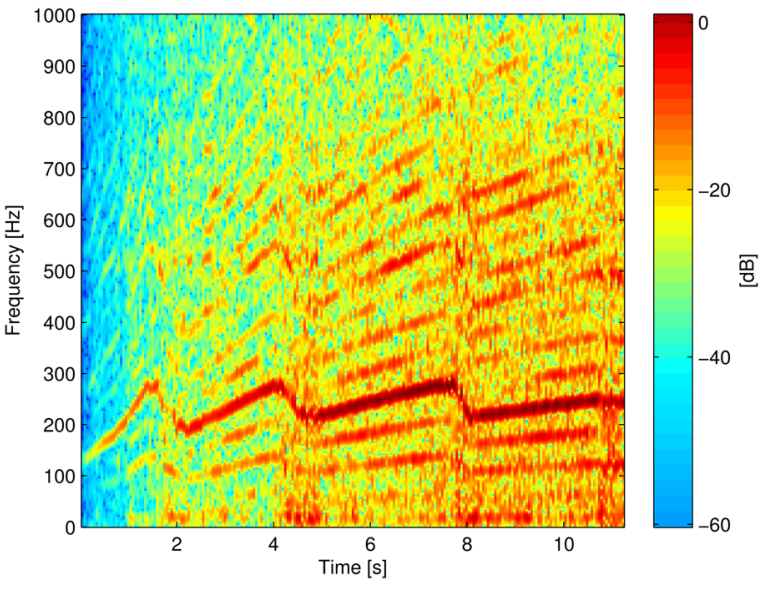

FIG. 5. (Color online) Spectrogram of an accelerating car.

sources. The setup is assumed to be in a free field and there is no feedback from the secondary sources to the reference sensor. A sample frequency of $f_{s}=2000 \mathrm{~Hz}$ was chosen. The goal of the system is to minimize the error at sensors $e_{1}$ and $e_{2}$ by choosing the appropriate filters $\mathbf{W}_{\mathrm{FIR}, i}^{(1,1)}(z)$ and $\mathbf{W}_{\mathrm{FIR}, i}^{(1,2)}(z)$. A regularization coefficient $\delta$ which is nearly optimal was chosen, which means that a fast rate of convergence is achieved without having an overshoot at convergence. The forgetting factor was set equal to $\lambda=1$. Since a symmetrical setup is used, two identical convergence curves are expected and simulation results show exactly this behavior as can be seen in Fig. 4. The filter is turned on after 1000 samples. The convergence curves of Fig. 4 are the result of averaging 50 simulations.

\section{B. Changing spectrum}

The ability of the algorithm to track changes in the spectrum of the noise source was tested with a similar setup, as shown in Fig. 3. An audio file of an accelerating car was chosen as noise source with a varying spectrum as shown in Fig. 5. The audio-file was filtered with an anti-aliasing filter beforehand to accommodate for the sampling frequency of $f_{s}=2000 \mathrm{~Hz}$. The algorithm was turned on after $1 \mathrm{~s}$ and the
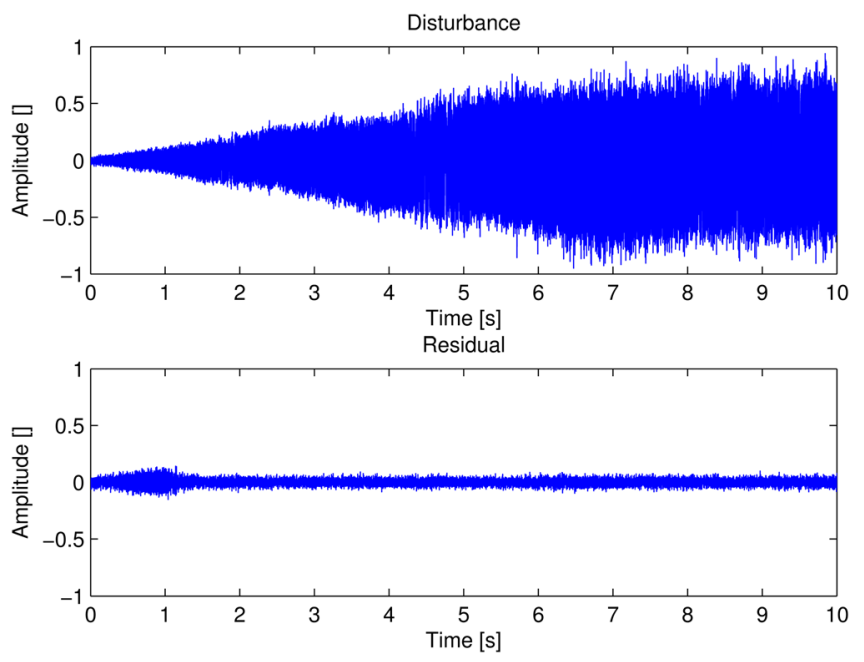

FIG. 6. (Color online) Disturbance and residual after active control.

S. van Ophem and A. P. Berkhoff: Multi-channel Kalman filters 


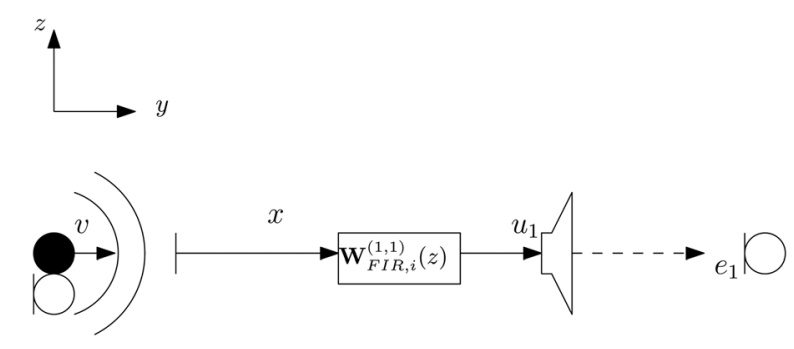

FIG. 7. SISO setup for moving noise sources.

result is plotted in Fig. 6. It can be seen that the algorithm has no difficulty with following the changing spectrum.

\section{Moving noise source}

This section presents simulation results for a moving noise source in which the primary path changes. A factor which can reduce the performance of the system is the Doppler effect, which can cause a significant shift in the measured frequency at the reference sensor compared to the error sensors if the noise source velocity is high. The Doppler effect was introduced into the simulations with the equations as shown in Ref. 13. The robustness of the SISO version of the algorithm to secondary path modeling errors is already investigated by Ref. 5. The other non-stationarities were tested in separate simulations. For simplicity a SISO setup was used in free field conditions, as shown in Fig. 7.

\section{Tracking behavior of primary path changes}

The influence of a changing primary path was tested with the following simulation: A noise source was assumed to be moving along the $y$-axis whereas the error sensor and secondary source had fixed positions on the $y$-axis. A reference sensor was co-located with the noise source. This setup gives a constant secondary path but a changing primary path. The velocity was set at $v=0.01 \mathrm{~m} / \mathrm{s}$ so that a negligible Doppler shift occurs. In Fig. 8 the results are plotted for a white noise source. The filter is turned on after $0.5 \mathrm{~s}$. After filter convergence the residual signal becomes progressively

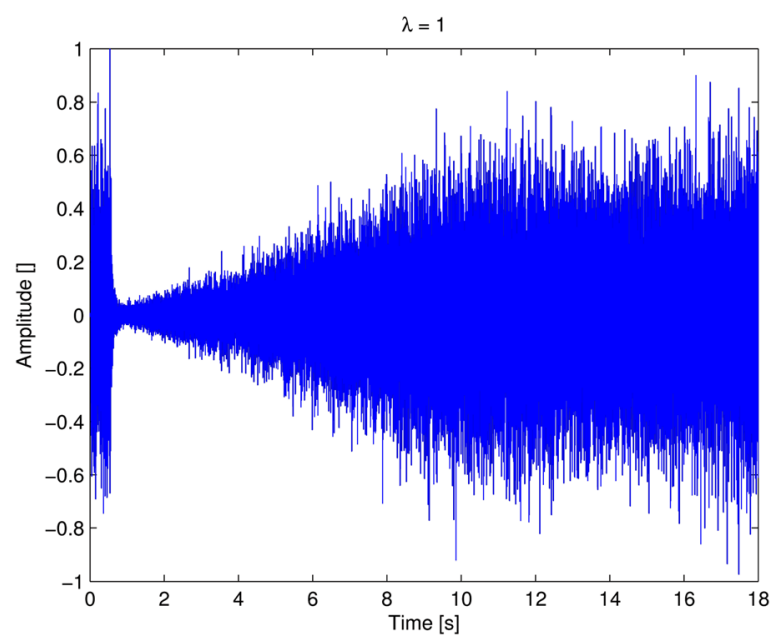

FIG. 8. (Color online) Noise source moving at $v=0.01 \mathrm{~m} / \mathrm{s}$, the filter is turned on after $0.5 \mathrm{~s} ; \lambda=1$. larger as a function of time. It can be concluded that the filter has a poor tracking performance. Since the filter estimation part has been formulated as an exponentially weighted RLS filter to increase tracking, a forgetting factor lower than 1 was tried.

Although an improvement in tracking was achieved, the algorithm becomes unstable after a number of iterations depending on the value of $\lambda$. This is shown in the upper part of Fig. 9. Instabilities were also observed when a forgetting factor is used in Eq. (33). These instability problems are known for the exponentially weighted fast RLS algorithms but although some suggestions for improving the numerical behavior are done in Refs. 8, 14, and 15, no solutions preventing instability for the fast array RLS form are known as of yet. Sayed ${ }^{8}$ suggests that possible numerical problems occur in the hyperbolic rotation needed for the calculation of $\Theta_{i-1}$. Multiple implementations for calculating the transformation matrix have been tried, including one Householder transformation, a combination of a circular Givens rotation with a hyperbolic Givens rotation, and the orthogonal diagonal method. It was observed that all these variants have different numerical behavior but the instability occurs with every method. For comparison the array version of the Kalman filter without the built-in shift-invariance property was tried. ${ }^{8}$ This algorithm uses only circular transformations, and it was found that the algorithm does not suffer from the mentioned instability, as shown in the lower part of Fig. 9 but it needs significantly more arithmetic operations per iteration, making it less suitable for real-time implementations.

\section{E. Experimental results}

A SISO version of the algorithm was implemented on the real time control platform, using Xenomai Linux, as described in Ref. 16. The algorithm was tested in an experiment in which the goal was to minimize the acoustic
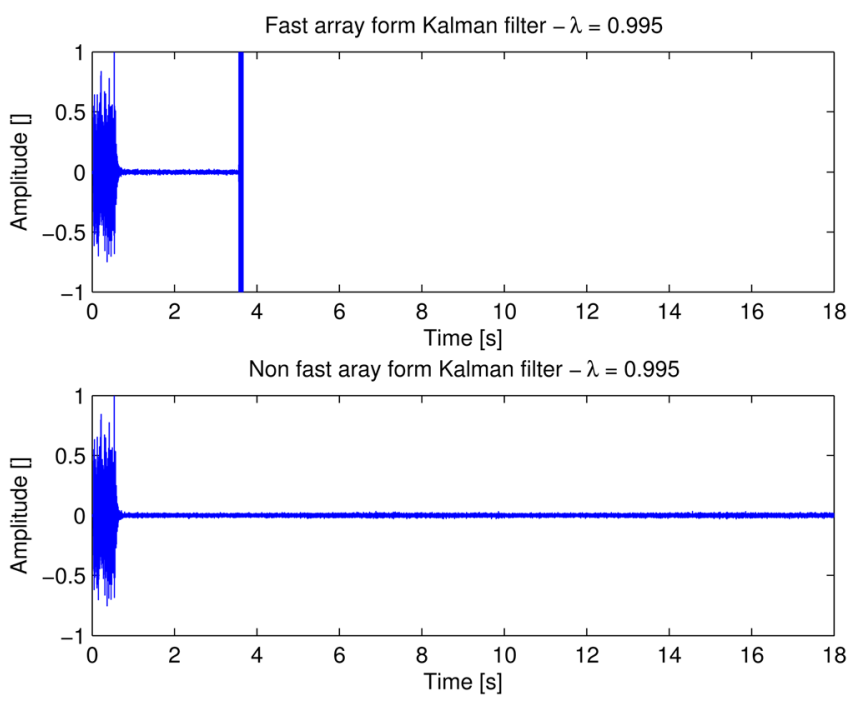

FIG. 9. (Color online) Noise source moving at $v=0.01 \mathrm{~m} / \mathrm{s}$, the filter is turned on after $0.5 \mathrm{~s} ; \lambda=0.995$. The upper part of the figure shows the unstable behavior of the fast array form of the Kalman filter. The lower part shows the performance of the same Kalman filter without the built in shiftinvariance and here the instability does not occur. 
pressure at the right-hand side of a duct, caused by a white noise source on the left-hand side of a duct. The duct has a length of $3.10 \mathrm{~m}$. At $45 \mathrm{~cm}$ from the end of the duct a secondary noise source was placed. The duct was placed in a lab environment of dimensions $4.50 \times 5.20 \times 3.50 \mathrm{~m}$. At the end of the pipe a microphone was placed and a digital reference signal was used, which is the same signal driving the white noise source at the end of the duct, so that no feedback from the secondary loudspeaker to the reference signal occurs. A sample rate of $f_{s}=2000 \mathrm{~Hz}$ was chosen and an FIR filter with $n_{w}=150$ filter coefficients was used. The order of the secondary plant model was chosen as $n_{s}=30$ and system identification was done off-line with sub-space identification, using SLICOT libraries. The variance accounted for values for this order were approximately $99.8 \%$.

\section{Influence of different secondary path models}

Implementing the full state space model in the algorithm did not lead to satisfactory results. At a sample rate of $2000 \mathrm{~Hz}$ the maximum processing power of the platform was exceeded, so another experiment was done with a sample rate of $500 \mathrm{~Hz}$. At this sample rate the available processing resources were sufficient but still the algorithm did not work properly. In order to reduce the influence of round-off errors and improve calculation efficiency, an output normal form parameterization as described in Sec. III was used for the state space description of the secondary path. This parameterization resulted in a good working implementation of the algorithm. A sample rate of $2 \mathrm{kHz}$ was used. The convergence curve of an experiment and the power spectral density of the error signal before and after control are shown in Figs. 10 and 11. A reduction of the error signal of $14.1 \mathrm{~dB}$ was achieved.

\section{Stability}

With a forgetting factor of $\lambda=1$ the algorithm was found to be robust. During experiments the algorithm did not diverge after a running time of a few hours. In these experiments the transformation matrices were calculated with

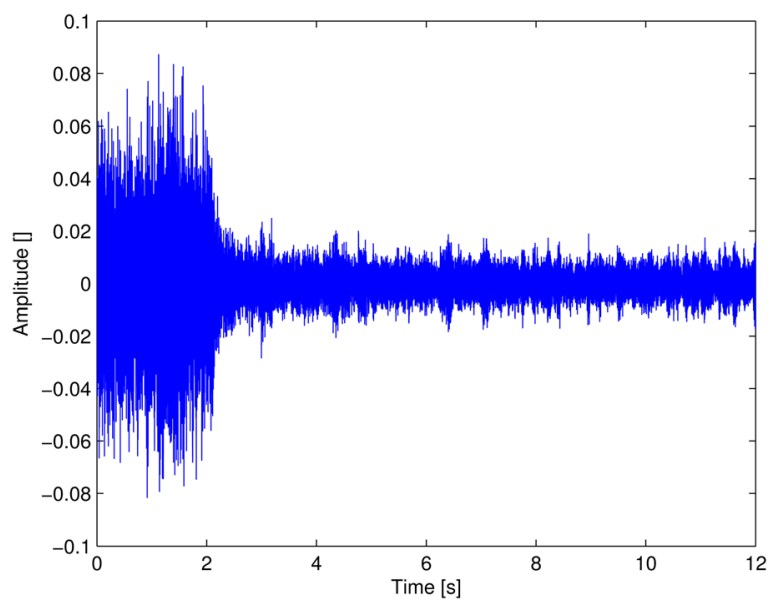

FIG. 10. (Color online) Amplitude measured at the error microphone; the filter is turned on after $2 \mathrm{~s}$.

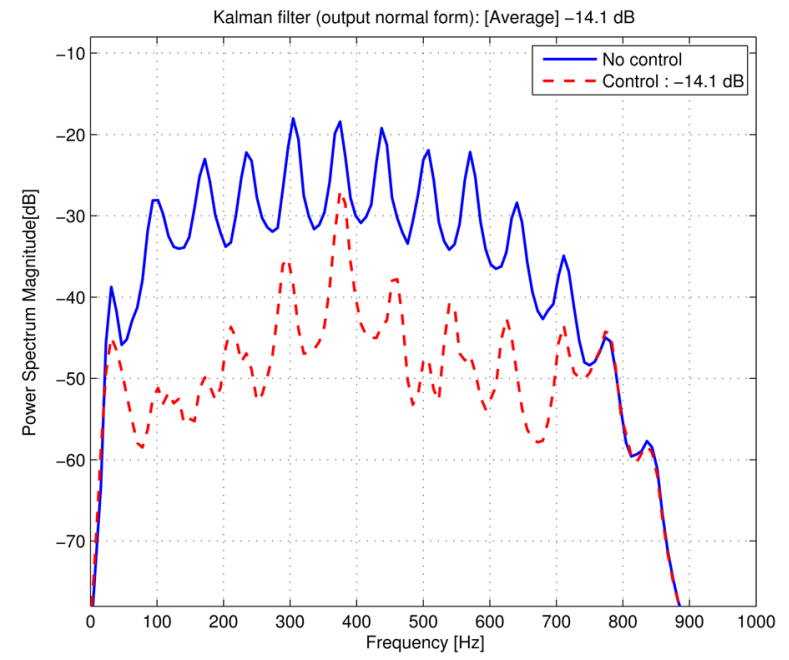

FIG. 11. (Color online) Power spectral density plot of the error signal before control and after control.

hyperbolic Householder transformations. In experiments with a lower forgetting factor the algorithm, i.e. the fast array version, diverged, which was as expected from the numerical simulations.

\section{CONCLUSIONS}

In this paper a MIMO fast array Kalman filter is derived for use in a feed-forward ANC system. It is shown how an output normal form parameterization of the secondary path can be implemented effectively into the Kalman filter equations. The performance of the filter was tested in both simulations and in a real-time environment in different noise control setups. It was shown that the filter achieves a good performance in the case of a constant primary path, both with a constant frequency spectrum and a changing frequency spectrum. When the noise source is moving, a good tracking performance can be achieved for a forgetting factor lower than 1 . In the fast array form of the Kalman filter numerical instabilities are observed for a forgetting factor lower than 1, contrary to the Kalman array form without an incorporated shift-invariance. Numerical results and experimental results correspond well with each other if the estimated secondary path is written in output normal form.

Additional research is needed to find a solution to the numerical instability of the fast-array form for forgetting factors smaller than unity.

\section{ACKNOWLEDGMENTS}

The authors would like to thank Henny Kuipers and GeertJan Laanstra for their technical support and André de Boer for giving the opportunity to do the initial part of this research. Part of this work was supported by TNO Built Environment.

${ }^{1}$ S. J. Elliott, Signal Processing for Active Control (Academic Press, London, 2001), Chap. 3.3.2, p. 124.

${ }^{2}$ E. Bjarnason, "Active noise cancellation using a modified form of the filtered-x lms algorithm," in Proceedings of Eusipco 92, 6th European Signal Processing Conference, pp. 1053-1056 (1992). 
${ }^{3}$ S. J. Flockton, "Fast adaption algorithms in active noise control," in Proceedings of the Second Conference Recent Adv. Active Control Sound Vib., pp. 802-810 (1993).

${ }^{4}$ A. H. Sayed and T. Kailath, "A state space approach to adaptive RLS filtering,” IEEE Signal Process. Mag. 11, 18-60 (1994).

${ }^{5} \mathrm{R}$. Fraanje, "Robust and fast schemes in broadband active noise and vibration control," Ph.D. thesis, University of Twente (2004).

${ }^{6}$ K. Zhou, J. C. Doyle, and K. Glover, Robust and Optimal Control (Prentice Hall, NY, 1996), Chap. 2.5, pp. 25-26.

${ }^{7}$ B. Sayyarrodsari, J. P. How, B. Hassabi, and A. Carrier, "Estimation-based synthesis of $h_{\infty}$-optimal adaptive fir filters for filtered-lms problems," IEEE Trans. Signal Process. 49(1), 164-178 (2001).

${ }^{8}$ A. H. Sayed, Fundamentals of Adaptive Filtering (Wiley, NY, 2003), Chaps. 12-14, pp. 732-873.

${ }^{9}$ A. H. Sayed and T. Kailath, "Extended Chandrasekhar recursions," IEEE Trans. Autom. Control 39(3), 619-623 (1994).

${ }^{10}$ T. Kailath, A. Sayed, and B. Hassabi, Linear Estimation (Prentice Hall, Englewood Cliffs, NJ, 2000), Chap. E. 6, p. 786.
${ }^{11}$ M. Verhaegen and V. Verdult, Filtering and System Identification: A Least Squares Approach (Cambridge University Press, Cambridge, 2007), Chaps. 7.3.1-7.3.2, pp. 219-227.

${ }^{12}$ R. A. Roberts and C. T. Mullis, Digital Signal Processing (AddisonWesley, Reading, MA, 1987), Chap. 10.4, pp. 449-481.

${ }^{13}$ A. Dowling and J. E. F. Williams, Sound and Sources of Sound (Ellis Horwood, Chichester, 1983), Chap. 9, pp. 199-204.

${ }^{14}$ A. W. Bojanczyk and A. O. Steinhardt, "Stabilized hyperbolic householder transformations," IEEE Trans. Acoust., Speech, Signal Process. 37, 1286-1288 (1989).

${ }^{15}$ T. K. Moon, K. Hencke, and J. H. Gunther, "An approach to stabilizing the fast array rls adaptive filter using homogeneous coordinates in projective geometry," in Asilomar Conference on Signals, Systems and Computers-ASILOMAR, pp. 1006-1009 (2010).

${ }^{16}$ J. M. Wesselink and A. P. Berkhoff, "Fast affine projections and the regularized modified filtered-error algorithm in multichannel active noise control," J. Acoust. Soc. Am. 124(2), 949-960 (2008). 